\title{
JUDAS ISCHARIOTH
}

\section{IN LEGENDE IND SAGE DES MITTELALTERS.}

$I_{11}$ durchaus einfacher und schmuckloser rede erzählt die heilige schrift die gefangennahme und die kreuzigung Christi; sie lässt die tatsachen bloss durch sich selbst wirken. Gegen den verräter Judas lässt sie weder ein wort des tadels laut werden, noch bemüht sie sich, seine handlungsweise zu motivieren. Lm so melr spielraum war hier der phantasie des mittelalters dargeboten. Auf der einen seite wurde die erzählung der bibel selbst durch allerlei kleine zigge vermehrt, auf der andern wurden die spärlichen nachrichten, die wir uber Judas besitzen, zu einer vollständigen biographie ergänzt; ausserdem bemühte man sich, wie wir sehen werden, die höllenstrafen des Judas möglichst schauerlich auszumalen.

Was nun zunächst die direct an die bibel anschliessenden traditionen betrifft, so muste vor allen dingen die legende bei dem bestreben nachlelfen, einen grund furr den verrat aufzufinden. In der bibel war bekanntlich bloss erzählt, Judas sei zu den hohenpriestern gegangen und habe ihnen angeboten, Christum zu verraten. Das ein!zige nachteilige, das vorher von Judas erzählt wird, ist, dass er, als Christus in Bethanien bei Lazarus war, Maria getadelt habe, weil sie sich zur salbung Christi einer, wie er sagte, allzukostbaren salbe bediente. ${ }^{1}$ ) Judas soll den verkauf der salbe um 300 groschen angeraten haben, wie er selbst sagte, um diese sunme unter die armen

1) Joh. 12. 4-6. Jedoch ist Johannes der einzige, der diesen vorwurf speciell dem Judas in den mund legt; Marcus (14.4) und Mattliäus (26. s) driicken sich allgemein aus.

Beiträge zur geschichte der deutschen sprache. 1 . 
zu verteilen, in wirklichkeit aber, weil er sie unterschlagen wollte. Diese begebenheit wurde nun mit dem verrat in zusammenhang gebracht. Es entstand die tradition, Judas habe als kassenfuhrer von allen einnahmen ein zehntel sich angeeignet; um sich nun für den verlust der 300 groschen schadlos zu halten, habe er Christum für 30 groschen verkauft. 1) Diese tradition steht aber in directem widerspruch mit der heiligen schrift, welche bei der erzählung der scene im hause des Lazarus von 300 groschen (denaren), bei angabe des ver-

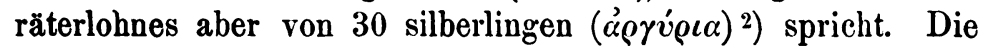
'Towneley-mysteries, in welchen Judas in einer langen rede vor dem synedrium auseinandersetzt, dass er Christum aus dem angegebenen grunde verraten wolle, sprechen von 300 pfund. ${ }^{3}$ ) - Die dreissig silberlinge, welche den preis des verrates bildeten, wurden selbst der mittelpunkt einer eigentümlichen und vielverbreiteten sage. Sie zeigt, wie alle gelehrten sagen, die tendenz, den gegenstand bis zu seinen z̈ussersten anfängen zurück zu verfolgen. Es wurde gefabelt, die silberlinge hätten ursprünglich Tharah, dem vater Abrahams gehört und Abraham hätte sie zum ankaufe des familiengrabes verwendet. Wenn die sage so weit zurückgriff, so liess sie sich naturlich auch nicht die gelegenheit entgehn, die 30 silberlinge als lohn fur den verkauf Josephs zu bezeichnen; es lag ja sehr nahe, diesen verrat mit dem verrat Christi in zusammenhang zu bringen, da derselbe zur erhöhung Josephs und zur rettung der Israeliten aus der hungersnot ebenso notwendig

1) In dem mittelniederländischen osterspiel (mitgeteilt von Zacher in Haupts zeitschrift II, 346) wird die sache so dargestellt, dass Judas als kemerere rechtmässig einen anteil von einem zehntel gehabt habe.

2) Matth. 26, 15.

3) Towneley-Mysteries. London 1836 für die Surtee-Society. p. 176 f. Die legenda aurea sucht auch die verschiedenen versionen zu vereinigen. Sie sagt bei erzählung der legende von Judas (p. 184-86 ed. Graesse): Dolens vero tempore dominicae passionis, quod unguentum, quod trecentos denarios valebat, non fuerat venditum, ut illos etiam denarios furaretur, abiit et dominum triginta denariis vendidit, quorum unusquisque valebat decem denarios usuales et damnum unguenti denariorum recompensavit; vel, ut quidam ajunt, omnium, quae pro Christo dabantur, decimam partem furabatur et ideo pro decima parte quam in unguento amiserat, scilicet pro triginta denariis, dominum vendidit. 
var, wie der verrat des Judas zur erlösung der menschheit (durch Christum. ${ }^{1}$ ) Die geschichte der silberlinge bis zur zeit (Yhristi wurde durch Gottfried you Viterbo poetisch behandelt. ${ }^{2}$ ) Auch in der legende vom heiligen Orendel kommen die silberlinge vor; es wird dort erzählt, dass sie der preis waren, um welchen Orendel dem fischer den heiligen rock Christi abkaufte.

Was nun den weiteren verlauf der passion betrifft, so bemühte man sich, überall etwas ungünstiges gegen Judas herauszulesen, auch da, wo die heilige schrift nicht einmal von ihm spricht. Besonders stark ist darin Abraham a Santa Clara in seinem grossen werke 'Judas der erzschelm'. ${ }^{3}$ ) Da er in diesem umfangreichen buche bestrebt ist, alle möglichen sünden und verbrechen an dem beispiele des Judas darzustellen und zu erläutern, so kommt es ihm gar nicht darauf an, eine stinde, welche weder die bibel noch die tradition von Judas berichtet, demselben einfach anzudichten oder, wenn der ausdruck erlaubt ist, anzuinterpretieren. So folgert er daraus, dass Judas Christum zum jüngern hohenpriester, Kaiphas, anstatt zum ältern, Annas, führte, Judas habe keine ehrfurcht vor dem alter gehabt. ${ }^{4}$ ) Bei erzählung der fusswaschung sagt er, Judas werde sich jedenfalls bei derselben den ersten platz angemasst haben und knüpft hieran eine längere auseinandersetzung tuber die grobheit.5) Für unsern zweck ist das werk Abrahams dadurch von besonderer bedeutung, dass der verfasser im laufe der darstellung sehr oft mit grosser belesenheit stellen aus älteren kirchenschriftstellern citiert, welche deutlich zeigen, wie viel die willkürliche interpretation der heil. schrift

1) Ueber diese typologische aufrassung der erzählung von Josephs verkauf vgl. Isidurus, allegoriae sacrae scripturae. Ausg. von Arevalo vol. V pag. 125.

2) Abgedruckt bei Du Méril, poesies populaires latines du moyen âge pag. $321 \mathrm{ff}$. Ueber ein denselben geyenstand behandelndes catalonisches gedicht ef. Milà y Fontanals in Eberts jahrbuch für romanische und exglische literatur $\mathrm{V}$, pag. 137 anm.

э) Judas der Erzschelm für ehrliche Leuth oder eigentlicher Entwurff und Lebensbeschreibung dess Ischariothischen Bösewichts etc. vol. I, I, III. Bonn 1687, vol. IV Salzburg 1695.

4) vol. IV pag. 263.

s) vol. II pag. 215. 
dazu beitrug, dass sich allmählich sagenhafte züge an die person des Judas ansetzten. Gerade bei besprechung der stelle der bibel, welche berichtet, wie sich Judas den hohenpriestern zur verfügung stellt ${ }^{1}$ ), wird einer sehr eigentümlichen interpretation gedacht. Judas sagt nämlich an der betreffenden stelle: 'Was wollt ihr mir geben? ich will ihn euch verraten.' Daraus, dass Judas hier Christum nicht ausdrücklich mit namen nennt, wird nun gefolgert, der teufel habe ihm ron dem momente an, wo der plan zum verrate in ihm auftauchte, die zunge gebunden, so dass er den heiligen namen nicht mehr aussprechen konnte. ${ }^{2}$ )

In den geistlichen spielen ist die auffassung des Judas bei der darstellung dieser scene vielfach sehr eigentümlich. Es entwickelt sich nämlich nicht selten bei dieser gelegenheit eine komische scene, indem Judas mit den hohenpriestern um den preis des verrates schachert. ${ }^{3}$ ) In ähnlicher weise sind auch manchmal bei der schilderung des teufels, der mitglieder des hohen rats, der behüter des heiligen grabes und anderer feinde Christi komische züge angebracht. In dieser komischen darstellung der feinde des christentums ist das freudige bewustsein ausgedrïckt, dass allen gegnern zum trotz Christus dennoch schliesslich den sieg errang, dass also alle feindlichen machinationen im grunde doch keinen nachteiligen erfolg hatten.

An eine ausführlichere ausschmückung des abendmahls scheint sich die legende nicht gewagt zu haben. Thévenot erzählt allerdings in der beschreibung seiner reise in den orient ${ }^{4}$, dass sich hier bei den koptischen christen eine eigentimliche tradition erhalten habe. Diese erzählen nämlich, dass an tage des abendmahls ein gebratener hahn auf dem tische ge standen habe. Als sich nun Judas entfernte, habe Christus den hahn wider lebendig gemacht und ihm befohlen, den Judas nachzufolgen. Der hahn tat dies und berichtete Christs

1) Matth. 26, 15 .

$\left.{ }^{2}\right)$ vol. II pag. 130. Abraham citiert hier 'Euthymius in Marcum'.

3) vgl. Wilken, Geschichte der geistlichen Spiele in Deutschlanc, pag. 183. Ausserdem Weigand iiber die Friedberger passion in Haupts zeitschrift VII, 549.

4) Thévenot, Rélation d'un vưage fait au levant pag. 501. 
alsdann, dass Judas ihn verraten habe und fur diesen dienst, den der hahn dem erlöser erwies, wurde er in das paradies versetzt. Der letztere umstand, dass ein tier in das paradies versetzt wird, beruht offenbar auf mohammedanischem einfluss; in dem paradies der Molrammedaner befindet sich bekanntlich ja auch der esel, auf welchem Christus in Jerusalem einritt. Dass Christus übrigens schon vor dem abendmahle Judas nicht traute, will Abraham a Santa Clara daraus schliessen, dass er sich einmal, als er sich nach dem einkauf von lebensmitteln erkundigen wollte, an Philippus und nicht an Judas wandte, der doch eigentlich als kassenführer dafür hätte sorgen müssen.1) Da aus der bibel ${ }^{2}$ ) hervorzugehen scheint, dass der tcufel während des abendmahles in Judas gefahren sei, so suchte man diesen vorgang in vielen mysterien bei der abendmahlscene dadurch zu versinnlichen, dass sich Judas einen schwarzen vogel vor den mund hält und flattern lässt, als ob derselbe ihm in den mund hineinflöge. ${ }^{3}$ )

Bei der schilderung der scene im garten Gethsemane halten sich die mysterien meistenteils streng an den text der bibel, der an dieser stelle ganz besonders zur dramatischen behandlung geeignet ist. Der kuss des Judas wurde von Origenes dadurch motiviert, dass Christus verschiedene gesichter annehmen konnte, von andern dadurch, dass Christus sonst vielleicht mit dem ihm sehr ähnlichen Jacobus minor verwechselt worden wäre. ${ }^{4}$ )

Der selbstmord wurde Judas im mittelalter fast zu einem moch grösseren verbrechen angerechnet, als der verrat, einmal veil das mittelalter den selbstmord als ein ganz besonders unnaturliches verbrechen verabscheute, dann aber auch deg-

') vol. I, pag. 314 .

2) Joh. 13, 2i.

$\left.{ }^{3}\right)$ cf. Mone, Deutsche Schauspiele des Mittelaiters II, 258. Auch in cen bildwerken treffen wir diese vorstellung an, cf. Heider, Beiträge 7ur christlichen Typologie aus Bilderhandschriften des Mittelalters. rien 1861. Tafel IV. In hyzantinischen bildwerken wird mehrmals (argestellt, wie dem Jadas ein kleiner teufel ins ohr fliistert. Jameson, sacred and legendary art. 3. aufl. London 1866. I, 258.

1) Judas vol. III, 266 u. 26i. Daraus, dass Jesus sich zum kusse reigte, schliesst Abraham, dass Judas von kleiner gestalt war. 
halb, weil man in dem selbstmorde des Judas ein frevelhaftes verzweifeln an der göttlichen gnade erblickte.1) Merkwürdiger weise aber soll sich nach einigen Judas deshalb erhängt haben, damit er noch vor Christi höllenfahrt in der hölle ankäme; weil er hoffte, dass er alsdann auch in anbetracht seiner reue von Christo aus der hölle gerettet werde. ${ }^{2}$ )

Bei der erzählung und darstellung von Judas tode bemuhte man sich vor allen dingen, den an dieser stelle in der bibel scheinbar obwaltenden widerspruch auszugleichen. Matthäus nämlich erzählt, Judas sei bald nach der gefangennahme Christi von reue ergriffen worden, habe das geld, das er fur den verrat empfangen hatte, im tempel hingeworfen und habe sich selbst erhenkt. ${ }^{3}$ ) In der apostelgeschichte aber erzählt Petrus, er sei von einer höhe (absichtlich oder unabsichtlich?) herabgestirzt und habe sich den leib aufgerissen, so dass die eingeweide heraustraten. ${ }^{4}$ ) Luther tibersetzt allerdings fälschlich, er habe sich erhenkt. ${ }^{5}$ ) In den mysterien war die letztere version entweder einfach ignoriert oder es wird dargestellt, dass der strick reisst und Judas herabstürt. Das zerplatzen des leibes wurde auf der blihne vielfach mit widerlicher natürlichkeit dargestellt; es wurde dadurch motiviert, dass man annahm, die seele habe keinen andern ausweg aus dem leibe finden können; der weg durch den mund sei ihr versperrt gewesen, weil Judas Christum geklisst habe. Aeusserlich wurde

1) Dies wird auch in sehr vielen mysterien besonders hervorgehoben.

${ }^{2}$ ) Diese auffassung findet sich $u$. a. in einer koptischen legende ubers. von Dulaurier, vgl. Douhet, Dictionnaire des légendes du Christianisme $720 \mathrm{ff}$.

3) Matth. 27, 3-6.

1) Apostelgeschichte I, 18. Petrus sagt hier von Judas: $\pi \varphi \eta v \dot{\eta} s$

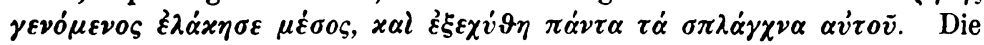
vulgata übersetzt: suspensus crepuit medius et diffusa sunt omnia viscera ejus.

5) Auch andere kleine variationen kommen in den mysterien vor; eine übersicht der theologischen versuche, den widerspruch dieser beiden stellen auszugleichen, findet sich in Herzog's realencyklopädie und bei Ersch u. Gruber s. v. Judas. Eigentümlich ist die erklärung von Lightfoot (Horae hebraicae in evangelium Matthaei zu XXVII, 5): Cum jam post projectos in templo proditionis suae nummos recederet ad suos reversurus, raptum eum in sublime diabolus, qui in ipso habitabat, strangulavit 
cilies auf der buhne dadurch dargestellt, dass Judas vorn in sieinem kleide einen vogel verbarg, welchen er bei der orhängungsscene fliegen liess.

Sehr oft wurde der tod des Judas auch als eine förmliche hinrichtung durch den satan dargestellt. ${ }^{1}$ ) Der teufel stieg Judas auf der leiter voran und zog ihn am stricke nach. Jedoch sscheint diese scene manchmal gar zu natürlich ausgefallen zu sein; ein Metzer chronist erzählt, dass Judas einmal bei (ller auffuhrung beinahe erstickt wäre. ${ }^{2}$ ) Deshalb scheint man sich häufig damit begnügt zu haben, eine puppe anstatt des Judas zu erhängen, wenigstens wird dies in der buhnnenanweisung für das passionsspiel des Frankfurter St. Bartholomäusstifts $^{3}$ ) ausdrücklich erwähnt. In dem Donaueschinger passionsspiel wird dargestellt, wie der teufel nach der erhängung mit .Judas in die hölle versinkt. Es wurde dies dadurch bewerkstelligt, dass beide sich an einem seile herabliessen, welches vom baume bis in die hölle führte. ${ }^{4}$ )

Ueber den baum, an welchem sich Judas aufgehängt thaben soll, gibt es verschiedene volkstümliche traditionen. Sehr verbreitet ist die sage, welche das zittern der espe davon herleitet, dass Judas sich an einer espe erhängt habe. ${ }^{5}$ ) Frisch gibt an, dass eine art des wilden johannisbeerbaums als der Judasbaum bezeichnet werde. ${ }^{6}$ ) In der Christiade des spanischen dichters Diego de Hojeda - welcher auch erzählt,

et praecipitem dedit, ita nt ad terram allisns rumperetur medius atque effunderentur ilia et tam horrendo exitu egrederetur diabolns.

1) cf. Mone, Schauspiele II, 281 ff.

2) Jubinal, mystères inédits pag. XIIIII.

3) Fichard, frankfurtisches Archiv für ältere deutsche Literatur und Geschichte. Ordnung des Passionsspiels der St. Bartholomäusstiftschnle zll Frankfurt a. M. 3. Theil pag. 14s.

4) Mone 1. c. Warum Judaz zwischen erde und himmel schweben muste, wird bei Jacobus a Voragire und im Passional (ed. Hahn pag. 318) auseinandergesetzt.

5) In loves lahours lost erwährt Shakspere noch eine andere tradition (act V, sc. 2). Dort sagt Birol, Judas sei an einem hollunderbaum (elder) erhängt worden, was gelegenheit $\mathrm{zu}$ einem wortspiele zwischen elder $=$ hollunder und elder $=$ älter gibr (cf. Delius, Shakspere I, 266 anm. no. 123). 'T'schischwitz, Nachklïnge germ. mythe in den werken Shakspeares s. $41 \mathrm{ff}$.

${ }^{\circ}$ Frisch, teutsch-lat. Wörterbuch L pag. 493. Art. Judasbaum. 
dass satan den Judas erhängt habe - ist von einem feigenbaum die rede. ${ }^{1}$ ) In manchen gegenden wird auch die weide als der baum bezeichnet, an welchem Judas sich erhängt habe. ${ }^{2}$ ) Im tiroler volksglauben ist es die weinrebe. Nach einer sehr ansprechenden vermutung, die mir herr professor Adalbert Kuhn gütigst mitgeteilt hat, läge hier eine verwechselung von wîde und ital. vite vor. In den mysterien habe ich nirgends einen bestimmten baum erwähnt gefunden.

Es sei gestattet, hier auch ein allerdings verhältnismässig sehr spätes geistliches schauspiel zu erwähnen, in welchem gleichfalls der selbstmord des Judas in sehr eigentämlicher weise geschildert ist. Es ist verfasst vou Christian Dedekind der gegen ende des siebzehnten jahrhunderts hofcomponist in Dresden war. ${ }^{3}$ ) Als Judas sich anschickt, sich zn erhenken, ruft ihm der teufel höhnend im echo seine letzten worte nach. Das zerplatzen des Judas und die böhnischen freudenbezeigungen des teufels machen einen halb widerliohen, halb komischen eindruck. ${ }^{4}$ )

Bei den volkstümlichen festlichkeiten, welche sich an die osterfeier anschliessen, spielt Judas natürlich auch eine grosse rolle. In Baiern wurde an mehreren orten eine puppe verbrannt, welche den Judas vorstellen sollte; die asche wurde alsdann ins wasser gestreut, weil man glaubte, dass sonst sturm und gewitter entstehen würde. ${ }^{5}$ ) Mehrfach wird auch

') Fray Diego de Hojeda, La Christiada VII, pag. 455, Biblioteca de autores españoles. vol. XVII. Madrid 1831.

$\left.{ }^{2}\right)$ Leoprechting, Aus dem Lechrain. München 1855.

$\left.{ }^{3}\right)$ vergl. Menzel, Deutsche Dichtung II, 223. Devrient, Geschichte der Ueutschen Schauspielkunst I, 277.

4) Ein gespräch der teufel an der leiche des Judas kommt auch in der passion des Jehan Michel vor, ef. Du Méril l. c. pag. 339. Auch in einem franzüsischen myster wird Judas bei dem selbstmorde von bösen geistern umgeben (Jameson sacr. and leg. art. I, 256, 57). Als er Christum vor Herodes stehen sieht, ergreifen ihn gewissensbisse. Hierauf erscheint die verzweiflung und bietet ihm werkzeuge zum selbstmorde dar. Er wählt den strick und hängt sich auf.

5) Ueber diese gebräuche cf. Panzer, Bayrische Sagen und Bräuche, Beitr. zur deutschen Mythologie 1, 212, wo dieser gebrauch aus dem Freisingischen erzählt wird und II, 530, wo bemerkt wird, dass er 
berichtet, um die osterzeit seien lärmende aufzüge gehalten worden, bei denen auch spottlicder gegen Judas gesungen worden seien. So fand in Lcipzig früher am sonnabend vor ostern ein unzug statt. Die kinder zogen mit pauken, schellen und klappern unter grossem geschrei in der stadt herum 'und sungen ein teutsches lied, welches dem verräter Juda zur schand und unehren von der geistlichkeit war gemachet worden.' 1) Ein ähnlicher gebrauch herschte in Zwickau. Dort fand am grünen dounerstag die sogenannte pumpervesper statt, 'da jedermann mit stecken, knüttelı, prügeln, steinen, hämmern, beilen - in der kirchen auf die stühle und bänke und wo es nur einen starken widerhall gab, schlug. Darbey muste der arme Judas viel leiden; jedermann redete alles übel von ihm und wollten ihn also zum teufel in die hölle jagen.' ${ }^{2}$ ) Auf diesen gebrauch bezieht sich offenbar cine stelle in Jörg Wickrams Rollwagenbtichlin. ${ }^{3}$ ) Dort ist die rede von 'Judas in der finstern metten; mit dem und uber den schreigt, singt unnd boldert man; wenig aber wirt dass leiden Christi bedacht.' Eine pumpermette erwähnt auch Schmeller, ohne dabei eines spottliedes auf Judas zu gedenken. ${ }^{\text {) }}$ In Köln singen auch die kinder am charfreitag ein Judaslied, das aber von Judas nichts mehr enthält. ${ }^{5}$ )

Es wurde also bei den osterfestlichkeiten häufig ein spottvers auf Judas gesungen. Es ist das offenbar das im mittelalter sehr verbreitete und oft parodierte Judaslied:

auch im Althennebergischen vorkommt und nachweise iber verwante sagen und gebriuche gegeben werden. In Böhmen wurden trither alljährlich am griindonnerstag alle confiscierten liberalen biicher zugleich mit der Judaspuppe verbrannt (Meissner, Rococobilder. Gumbinuen 1871, pag. 61).

1) Haltaus, Gilossar. Ait. Judasgruss.

2) Tobias Schmidius Chron. Zwickav. I, 374 bei Haltaus I. c.

3) ed. Heinrich Kurz. Leipzig 1565, pag. 85.

4) sichmeller, Bayrisches Wïrterbuch I, 1689 (2. ausg.)

5) L. Erk, Neue Sammlung deutscher Volkslieder vol. II, heft 6, pag. 44, cf. Soltau-Hildebrand, deutsche historische Volkslieder, zweites Hundert pag. 221. 
0 du armer Judas

Was hast du getan

Dass du deinen herren

Also verraten hast.

Darumb so mustu leiden

Hellische pein

Lucifers geselle

mustu ewig sein. ${ }^{1}$ )

Schon Hoffmann von Fallersleben vermutete, dass dieses lied aus einem alten osterspiele entnommen sei. Er hält das für um so wahrscheinlicher, als das volk bei derartigen spielen mitwirkte und als chor manches mitsingen muste. ${ }^{2}$ ) Durch eine stelle des oben erwähnten Frankfurter passionsspiels, von welchem uns allerdings bloss die buhnenanweisungen und die stichworte erhalten sind, wird diese vermutung auffallend bestätigt. Es wird nämlich dort angegeben, dass in dem augenblicke, in welchem Judas Christo den verräterkuss gibt, der chor (personae) einen gesang anstimmen solle, welcher beginnt: O Juda quid dereliquisti. ${ }^{3}$ ) Dies erinnert auffallend an den anfang unseres liedes. Das lied wurde namentlich im spätern mittelalter und in der reformationszeit sehr oft parodierend auf andere persönlichkeiten angewendet; Hildebrand und Hoffinann von Fallersleben haben hierfitr mehrere beispiele gesammelt; die in Haltaus glossar vorkommenden beispiele werden aber von beiden nicht erwähnt. Berlihmt ist die parodie dieses liedes, mit welcher Luther seine schrift gegen Heinrich von Braunschweig beschliesst; mit dieser parodie steht es offenbar in beziehung, wenn sich Heinrich im jahre 1545 dariber beschwert, dass der torwächter von Wolfenbuttel ihm zum spotte die melodie des Judasliedes geblasen habe. ${ }^{4}$ )

Während nun die christen sich bemtihten, Judas uberall

') cf. Hoffmann von Fallersleben. Geschichte des deutschen Kirchenliedes. 2. Aufl. pag. 230. Bartsch vermutet, dass es auch mit diesem liede zusammenhängt, wenn im Parzival $(219,25)$ Judas als der 'arme Judas' bezeichnet wird, vgl. die ausg. von Bartsch I, pag, 230.

2) l. c. pag. 231 .

3) Fichard, Archiv III, pag. 147.

‘) Hoffmann, Kirchenlied pag. 232. Haltaus, art. Judasgruss. 
in ein $n_{i}$ g̈glichst ungtinstiges licht zu setzen, waren auf der andern seite die feinde des christentums bestrebt, den verrat als eine berechtigte und löbliche handlung hinzustellen; besonders soll dies bei der sekte der Kainiten der fall gewesen sein.1) Ganz eigentümliche traditionen uber Judas waren bei den Juden im mittelalter in umlauf, nach welchen Judas im interesse seiner glaubensgenossen ein schweres verbrechen auf sich lud, um Christum zu falle zu bringen. Diese tradition bildet das sujet des buches 'Toledoth Jeschu. Es ist von Wagenseil in der von ihm 'Tela ignea Satanae' betitelten sammlung jüdischer schriften gegen das christentum herausgegeben, hehräisch mit lateinischer übersetzung, ausserdem gibt Wagenseil auch eine ausführliche confutatio als anhang; ferner wird diese schrift von Eiscnmenger in seinem buche 'Entdecktes Judenthum' (s. 1. 1700, 2. ausg. Königsberg 1711) sehr ausführlich behandelt. ${ }^{2}$ ) Wagenseil sagt, man könne nirgends aus dem buche ersehen, aus welcher zeit und aus welchem lande es stamme. ${ }^{3}$ ) Als die frtiheste ihm bekannte erwähnung des Toledoth Jeschu citiert er eine stelle aus dem pugio fidei des dominikaners Raimundus Martini, welcher gegen ende des dreizehnten jahrhunderts lebte ${ }^{4}$ ); jedoch gesteht er selbst zu, dass diese version mannigfache kleinere abweichungen von seinem texte zeige und ausserdem erwähnt Raimundus Martini nicht ausdrucklich den titei Toledoth Jeschu. Auch Luthern war diese merkwürdige schmähschrift nicht unbekannt. ${ }^{5}$ ) Der

1) vgl. Fabricius. Codex apocryphus novi testamenti I, 352. Irenaeus contra haeres. cap. 35 . Die schrift von Goezins 'de cultu Judae proditoris' (Libeck 1i13) kenne ich uur aus den anfiihrungen bei Du Méril und in Ersch u. Grubers encyklopädie s. v. Judas Ischarioth.

2) Liber 'Toldos Jeschu, die vorletzte der in der sammlung publicisten schriften (hesonders paginiert, ebenso die confutatio). Eisenmenger, entdecktes Judentum vol. 1 .

3) Statim se prodit ultro libri vitium, dum nec autoris nomen, nec aetatem suam, nec unde prodierit, ullatenus commonstrat, sed omnino talis cernitur quales famosi libelli solent.

4) Raimundus Martini. Pugio fidei (Verteidigung des christentums gegen Judeu und Mohammedaner). ed. Carpzov, Leipzig 1687, mit anmerkungen von de Voisin pag. $362 \mathrm{fti}$.

5) Die betr. stelle mitgeteilt von Wagenseil in der confutatio pag. 45. 
inhalt derselben ist höchst eigentümlich und ich gebe deshalb im folgenden zunächst eine kurze tibersicht.

Im eingange wird die schon in den ersten jahrhunderten unserer zeitrechnung ron den feinden des christentums aufgebrachte verleumdung erwähnt, dass Jesus der uneheliche sohn der Maria und eines gewissen Pandera gewesen sei ${ }^{1}$ ), hierauf wird die geburt Jesu ausführlich erzählt. Als er herangewachsen war, wird nun weiter berichtet, verweigerte er einstmals einem mitgliede des hohen rates die herkömmliche ehrenbezeigung; dadurch und durch seine uneheliche geburt geriet er friih in übeln ruf. Nun strebte er danach, zauberkiinste zu erlernen, um sich wider angesehen und gefürchtet $\mathrm{zu}$ machen und er erfuhr, dass er dies erreichen könne, wenn er in das allerheiligste des tempels eindringe und den daselbst in einen stein eingegrabenen unaussprechlichen namen gottes (Schem Hamphorasch) sich einpräge. Diesen stein hatte man gefunden, als das fundament zum tempel gelegt wurde und hatte ihn später in das allerheiligste gebracht. Um nun aber zu verhindern, dass jemand eindringe und das geheimnis verletze, hatten einige weise männer zwei eherne löwen an die pforten des allerheiligsten gestellt, welche durch ihr gebrüll jeden, der aus dem allerheiligsten hervortrat, so in schrecken setzten, dass er den heiligen namen wider vergass. Jesus aber drang ein, schrieb das Schem Hamphorasch auf einen pergamentstreifen und machte alsdann einen schnitt in seinen körper, in welchen er den pergamentstreifen hineinsteckte. Nun konnten ihm die löwen nichts anhaben, er ging aus dem tempel heraus, nahm das Schem Hamphorasch wider hervor und prägte es sich ein. In folge dessen tat er nun wunder und erwarb sich einen grossen anhang. Die mitglieder des hohen rats verklagten ihn nun bei der königin Helena, welche aber Christo glinstig gesinnt war (offenbar eine erinnerung an die gleichnamige mutter Constantins). Christus wird nun vor die königin gerufen, tut aher vor dieser grosse wunder und wird in folge dessen wider freigesprochen. Die Juden beraten nun mit einander, ob sie nicht vielleicht auf eine andere weise gehandelt.

1) Hieriber wird gleichfalls in Wagenseils Confutatio ausfïhrlich 
Christum zu falle bringen könnten und es wird der vorschlag gemacht, dass irgend einer in das allerheiligste dringen und gleichfalls das geheimnis des Schem Hamphorasch stehlen solle. Ein mitglied des hohen rates ${ }^{1}$ ) namens Judas erbietet sich dies zu tun, weun die übrigen mit ihm die sünde übernehmen wollten. Er erschleicht nun das geheimnis ganz in der nämlichen weise wie Jesus. Hierauf tritt er ror die königin und erklärt, er habe ganz dieselbe gewalt wunder zu tun, die sie vorher an Christo bewundert habe. Dieser aber, um einen neuen beweis seiner wunderkraft zu geben, steigt vor der königin und allem volke in die luft empor; Judas spricht nun das Schem Hamphorasch aus und fliegt ihm nach. Hoch in den lüften entsteht nun ein heftiger kampf zwischen beiden, der lange hin und her schwankt, bis es Judas gelingt, Christum mit sich herabzureissen. ${ }^{2}$ ) Nun soll Christus festgenommen werden, es gelingt ihm aber, mit hilfe seiner jünger zu entrinnen; er begibt sich nun an den Jordan und beginnt dort wider wunder zu tun. ${ }^{3}$ ) Hierauf erbietet sich Judas, ihm nachzueilen und ihm das Schem Hamphorasch zu rauben und wirklich gelingt es ihm, als Christus einmal in tiefen schlaf versenkt war, das pergament aus seiner seite hervorzuzichen. Christus erwacht, ist über den verlust seiner wunderkraft in verzweiflung und fordert die juinger auf, ihn in ihre mitte zu nehmen, so dass ihn niemand sehen könne und alsdann nach Jerusalem zu ziehen, denn dort lioffte er seine wunderkraft wider crlangen zu können. Judas mischt sich unerkannt unter die jünger und fordert, sobald er in Jerusalem ankommt, die ratsmitglieder auf, eine starke wache am tempel aufzustellen; wenn dann Christus mit den jüngern käme, wolle er denselben fur die wache dadurch erkenntlich machen, dass or ror ilım niederfalle und ihn anbete. Auf diese weise wird

1) Im text steht: Es erhob sich ein weiser.

2) Juda animadvertens, non se valere tantum ut opera Jeschu exsuperare queat, corporis sui saceats humore eum proluit unde inmundi redditi, in terram prolapsi sunt, ob contractam impuritatem ambo usu Schem Hamphorasch privi, quoad abluerentur (Wagenseil pag. 13).

3) Jeschu celeriter ad Jordanem properat, cumque se abluisset ac purificasset, effert iterum Nonen ac priora repetit miracula (Wagenseil pag. 14). 
auch in der tat Christus verhaftet. Seine geiselung und verhöhnung wird hierauf ganz in ähnlicher weise wie im neuen testamente erzählt. Er wird nun zuerst gesteinigt und soll alsdann an ein kreuz geschlagen werden. Nun hatte er aber, als er noch im besitz des Schem Hamphorasch war, indem er seinen tod vorhersah, alle bïume und hölzer beschworen, dass sie ihm kein leid antun sollten und in folge dessen brachen alle kreuze unter ihm zusammen. Da holte Judas aus scinem garten einen kohlstengel von ungeheurer grösse und an diesem wurde Christus aufgehängt. Am abende wird nun Christus begraben; Judas aber holt den leichnam wider aus dem grabe heraus und trägt ihn in seinen garten, wo er ihn in dem bette eines flusses vergräbt. Als nun am morgen die jünger an das grab kommen und den leichnam nicht mehr finden, verbreiten sie das gerücht, Christus sei in den himmel emporgestiegen zu seinem vater. Als die königiu dies erfährt, ruft sie die mitglieder des hohen rates vor sich, macht ihnen vorwürfe, dass sie den wahren sohn gottes getötet hätten und gibt ihnen auf, die leiche binnen drei tagen wieder herbeizubringen, widrigenfalls sie sämmtlich den tod erleiden müsten. Sie suchen nun uberall den leichnam, bis endlich einer von ihnen, Rabbi Tanchuma, Judas in seinem garten sitzend trifft und ihn vom gebot der königin benachrichtigt. Nun wird der leichnam wider hervorgeholt und dadurch das gericht von der auferstehung widerlegt. Als Christus aus seinem grabe gezerrt wurde, gingen ihm am schädel die haare aus, und deshalb wird gesagt - tragen noch heute die christlichen geistlichen eine tonsur.

Von dieser darstellung unterscheidet sich die version des Raimundus Martini dadurch, dass sie den hauptfeind Christi ausdrücklich Judas Ischarioth kampf in der luft so, dass Judas Christum von der höhe herabgeworfen und ihm den einen arm zerschmettert habe. Die ganze abenteuerliche geschichte mit dem leichnam Christi lässt Raimundus Martini unerwähnt; wie er denn uberhaupt das ganze nur deshalb anfuhrt, um die ansicht zu widerlegen, als habe Christus auf eine slindhafte weise seine wunderkraft erlangt.

Dies eigentumliche machwerk, dessen in die augen fallende 
inneren widersprïche von Wagenseil und Eisenmenger ausführich dargelegt werden, ist für unsern zweck deshalb wichtig, weil es sich offenbar in manchen einzelnen punkten an die darstellung des neuen testamentes anlehnt. So befindet sich auch in dieser darstellung Judas eine zeit lang als verräter unter den anhängern Christi, welcher hier ebenso wie im neuen testamente durch eine heuchlerische bezeigung der verehrung 'seinen feinden kenntlich gemacht wird. Im höchsten grade merkwürdig aber ist in der erzählung der umstand, dass Jesus alle hölzer beschwört, ihm keinen schaden zu tun und Judas schliesslich dennoch eine pflanze herausfindet, an welcher derselbe aufgehängt werden kann. Conrad Hofmann hat schon einmal in einer kurzen notiz ${ }^{1}$ ) auf die auffallende ähnlichkeit hingewiessn, welche diese erzählung mit dem mythus vom tode Balders hat. Hier beschwört Frigg alle hölzer, dem Balder keinen schaden zu tun, Loki aber, dessen stellung unter den ubrigen göttern auch sonst an die stellung des Judas unter den aposteln erinnert, schnitzt aus einem zweige der bei der beschwörung vergessenen mistel den pfeil, mit mit welchem Balder getötet wird. ${ }^{2}$ )

Auch bei den Mohammedanern sind eigentümliche traditionen uber Judas im umlauf, welche offenbar von alten christlichen sagen beeinflusst sind, sich aber zunächst an eine stelle des koran anschliessen. Dort wird nämlich berichtet, Christus sei nicht gekreuzigt worden, sondern sei, kurz ehe dies geschehen sollte, von einem engel in die luft entfuhrt worden, an seiner stelle aber habe ein anderer den kreuzestod erlitten. Hieran knupfte sich nun die erzählung an, dass Judas in das haus eingedrungen sei, in welchem sich Christus befand, um ihn dem tode zu uberliefern. Hierauf - wird weiter erzählterhielt Judas plötzlich durch ein wunder ganz genau dieselbe gestalt wie Christus; diesen aber ergriff der erzengel Gabriel und entfuhrte ihn in den himmel. Die Juden drangen nun in das haus und als sie Judas saben, glaubten sie, er sei Christus, sie schleppten ihn also mit sich fort und kreuzigten ihn. Erst nachdem er tot war, erkannteu sie ihren irrtum. Gerock,

1) Conrad Hofinann in Pfeiffers Germania II, 48.

2) Gylfagynning cap. 49. 
welcher in seiner christologie ${ }^{1}$ ) des koran auch von dieser sage handelt, erinnert daran, dass die entfuhrung Christi durch die luft und die unterschiebung eines andern opfers auffallend an die sage von der opferung der Iphigenie erinnere; auch die ähnlichkeit dieser tradition mit der geschichte von der opferung des Isaak hebt er hervor.

In dem bisherigen verlaufe unserer untersuchung haben wir gesehen, dass man dem Judas ebenso wie dem Pilatus auch innerhalb des in der heiligen schrift gegebenen rahmens möglichst viele schlechtigkeiten anzudichten suchte, von denen die bibel nichts weiss. Während aber hier die autorität der bibel davon abschrecken muste, der lust nach phantastischer ausschmückung vollständig die zügel schiessen zu lassen, hatte man ganz freien spielraum bei der schilderung desjenigen teiles von Judas leben, welcher dem zeitraum, den das neue testament behandelt, voraufgeht; und von dieser freiheit wurde denn auch der ausgedehnteste gebrauch gemacht. Es war im mittelalter eine sehr phantastische legende von dem leben des Judas bis zu seinem eintritt unter die jünger Christi in umlauf; niederschriften derselben finden sich über das ganze abendland verbreitet. Da diese niederschriften, so viel ich weiss, noch nirgends ubersichtlich zusammengestellt sind, so will ich sie in dem folgenden aufzählen, soweit sie mir bekannt geworden sind. - Erst nach abschluss des vorliegenden aufsatzes kam mir die schrift von d'Ancona: la leggenda di Vergogna e la leggenda di Giuda zu gesicht. D'Ancona behandelt besonders ausfuhrlich und mit grosser gelehrsamkeit die mit der Judaslegende verwanten legenden, von den aufzeichnungen der Judaslegende selbst hat er mehrere unerwähnt gelassen. Nachträge zu diesem buche lieferte Reinhold Köhler ${ }^{2}$ ), dessen guite auch die vorliegende arbeit mehrere notizen verdankt.

1) C. F. Gerock, Christologie des Koran, pag. 5s und 59. Hier wird auch der zusammenhang dieser sage mit alten christlichen anschauungen betont. Die verschiedenen überlieferungen der mohammedanischen sage zeigen mannigfache variationen.

2) Jahrbuch für Rom. u. Engl. Lit. XI, $313 \mathrm{ff}$. 
Die älteste erwähnung der legende findet sich wol bei Jacobus a Voragine, welcher sic aus einer 'historia licet apocrypha' entnommen haben will.') Er erzählt sie in verbindung mit der legende rom apostel Matthias, welcher bekanntlich die durch den tod des Judas unter den aposteln entstandene licke ausfüllte. Von solchen gelcyentlichen darstellungen der legende, wie sie sich z. b. auch im deutschen passional und in dem passionsspiel des Jehan Michel finden, will ich jedoch im folgenden absehen und nur diejenigen niederschriften aufzählen, welche die legende als ein abgeschlossenes ganzes darstellen.

In eiver solchen abgeschlossenen darstellung findet sich unsere legende

\section{1) Lateinisch.}

a. Ein gedicht in leoninischen hexametern. vers 1: Dicta vetusta patrum jam deseruere theatrum. Abgedruckt in Mone's Anzeiger für Kunde der deutschen Vorzeit. 1838 pag. $532 \mathrm{fi}$; in Lu Mérils Poésies populaires latines du moyen âge pag. $326 \mathrm{ft}^{2}$ ) In vielen handschriften erhalten, worüber ef. עu Méril l. c. p. 325 .

b. Ein gedicht in leoninischen hexametern. vers 1: Cunctorum veterum placuere poemata multum. Erhalten in einer handscbrift des 15. jabrhunderts, ef. Leyser, Historia poetarum et poematum medii aevi, pag. 2125.

\section{2) Englisch.}

a. Ein gedicht in paarweis gereimten langzeileu, abgedruckt bei Frederick J. Furnivall, Early English poenıs and lives of Saints pag. $107 \mathrm{ff}$. (Zweiter teil der 'Transactions of the philological society 1862.) ${ }^{3}$ )

b. Ein strophisches gedicht, der haudschrift der 'Towneley mysteries als auhang beigegeben. Nach einer anm. des herausgebers added in a more moderu hand, apparently

1) Jacobus a liuragine, Legenda aurea ed. Grässe. pag. 15t-56.

$\left.{ }^{2}\right)$ Du Méril hat nachgewiesen, dass das gedicht direct im anschluss al dic erzäliung cies Jacubss a Voragine verfasst ist.

3) Beginnl: Judas was a liper brid, pat Ihesu solde to rode. 
about the commencement of the sixteenth century. ${ }^{1}$ ) Abgedruckt in der ausgabe fur die Surtee-Society London 1836 (besorgt von Dr. Raine und J. Gordon) pay. $328 \mathrm{ff}$. Judas wird hier selbst redend eingefuhrt. Vielleicht ein monolog, den Judas in irgend einem mysterium vor seiner erhängung hielt, vielleicht auch eine bänkelsängerballade. ${ }^{2}$ )

c. 'The unhappy Birth, wiched life and miserable death of that vile 'Irajtor and Apostle Judas, who for thirty Pieces of Silver betray'd his Lord and Master Jesus Christ. - Durhan printed and sold by Isaac Lune. ${ }^{3}$ )

3) Schwedisch.

Volksbuch von Judas Ischarioth, aus dem schwedischen ubersetzt vou H. 'Tamms. Algedruckt in v. d. Hagens Germania VI, pag. 144 ff. ${ }^{4}$ )

1) Die erste strophe lautet:
Alas, Alas and welaway
Weryd and cursyd I have been ay
I slew my father and syn belay
my moder der
And falsly after, I can betray
Myn awn mayster.

2) Dass eine derartige ballade derjenigen person, von welcher sie handelt, in den mund gelegt wird, kommt auch sonst vor. Vgl. z. b. die ballade von Titus Andronicus, abgedruckt in Percys Reliques of ancient english poetry und in der Shakspereausgabe von Delius II, $115 \mathrm{f}$.

ग) Diese version unserer legende, uber welche mir Richard Wulcker glitigst mitteilung machte, enthält mehrere abweichungen von der gewohnlichen uberlieferung. Namen und abkunft der eltern sind anders angegeben, als bei Jacobus a Voragine: The father of Judas was one Machabaeus, a worthy Merchant being of the Tribe of Issachar, who was betrothed to one Berenice, a beautiful and rich Maden living in Hierapolis; but when the nuptials were solemnized, after the Jewish Custom, he brought his spouse home to Joppa to his own habitation. Offenbar wird hier eine seestadt als wohnort von Judas vater genannt, um die aussetzung in das meer wahrscheinlicher zu machen. In dem lande Iscarioth wird Judas vom könig (nicht wie sonst von der königin) gefunden; wird später ratgeber des königs. - Die vorliegenden notizen sind aus den capiteluberschriften und der gereimten inhalts:ngabe entnommen. Die schrift selbst war mir nicht zugänglich.

1) Hier wurde zum schluss eine vergleichung der darstellung 
4) Catalanisch.

De Judes Escarioth e de la sua vida. Milá y Fontanals in seiner ubersicht der altcatalanischen dichter (in Eberts Jahrbuch fur romanische und englische Literatur vol. V, pag. 137) erwähnt ein werkchen in versen unter diesem titel, als in die epoche von Ramon Lull gehörig. Höchst wahrscheinlich liegt dieser dichtung auch unsere legende zu grunde ${ }^{1}$ ) (pag. 137 anm. 2).

5) Spanisch.

Ein drama des dichters Antonio de Zamora (starb 1730). Ticknor sagt von ihm: 'Seine schauspiele ... uber religiōse gegenstände sind fast lächerlich, ausgenommen der Judas Ischarioth, welcher zu viele greuel enthält, um zu belustigen.' Dies drama war mir nicht zugänglich, unter den in der Biblioteca de autores españoles abgedruckten dramen Antonio de Zamora's ist es nicht enthalten. Aus der bemerkung Ticknors, die oben erwähnt ist (Geschichte der schōnen Literatur in Spanien ubers. von Julius II, 76 u. 77) düfte wol hervorgehen, dass dies drama gleichfalls auf unserer legende basiert.

6) Deutsch.

Eine kölnisch-niederrheinische prosaische ubersetzung von Judas leben aus der legenda aurea in dem Seelentrost, ed. Pfeiffer in Frommanns Deutschen Mundarten, II, 291. - Eine selbständige, zusammenhängende deutsche version der legende ist mir nicht bekannt. Man kōnnte allenfalls den Judas des Abraham a Santa Clara hierher rechnen (s. oben), jedoch sind hier die einzelnen punkte der erzählung durch lange betrachtungen von einander getrennt. Auf pag. 84 citiert er am rande den Jacobus a Voragine als seinen gewährsmann.

der legende in dem volksbuche und bei Abraham a Santa Clara gegeben.

1) Der zeit uach wäre dies sehr wol möglich, da Jacobus a Voragine 1298 and Lullus 1315 starb. 
7) Französisch.

Line altfranzösische bearbeitung in achtsilbigen reinpaaren, abgedruckt bei d'Ancona l. c. Eine bearbeitung in prosa findet sich in der bibliothèque Bleue, rgl. Douhet Dict. s. v. Judas.

8) Italienisch.

Die italienischen bearbeitungen sind von d'Ancona ausfuhrlich besprochen, ein italienischer text ist von ihm abgedruckt. Er spricht auch von einem irrtümlich dem Tasso zugeschriebenen gedichte: la dispcrazione cie Giuda (pag. 97).

Der inhalt der legende, deren verschiedene niederschriften bis auf ganz unbedeutende variationeu im wesentlichen tibereinstimmen, ist ungefähr folgender:

Cyborea, die gemahlin des Ruben aus dem stamme Dan, hat vor der geburt ihres sohnes Judas böse vorahnungen. In folge dessen wird derselbe, sowie er zur welt kommt, in einem kästchen auf dem meere ausgesetzt. Das kästchen treibt nuu an die insel Skarioth ${ }^{1}$ ) und die kinderlose königin dieser insel nimmt Judas als ihren sohn an, indem sie ihrem gemahl vorspiegelt, er sei ihr leibliches kind. Bald darauf bekommt sie aber selbst einen leiblichen sohn, der nun mit Judas zusammen erzogen wird. Da ihn aber Judas fortwährend mishandelt und beleidigt, entdeckt die königin in ihrem unmut ihrem gemahl, dass Judas bloss ein untergeschobenes kind sei. Judas ist

1) 'Denn welcher hangen sollte, konnte nicht ersaufen', hemerkt das schwedische volksbuch, ähnlich Abraham a Santa Clara I, 10. - Eine lundschaft $\Sigma x \alpha \rho / \alpha$ gibt es auf der insel Corfu und oxistieren in der tat volkstïmliche traditionen, wonach Judas in der dortigen gegend geboren sein soll. Pietro della Valle (Viaggi, Venezia 166i I, 4) sah dort noch einen mann, welcher fiir einen nachkommen des Judas galt. Johannes Brompton gibt ein Castellum desertum quod dicitur Butestic als geburtsort des Judas an (Twysden, Historiae Anglicanae scriptores

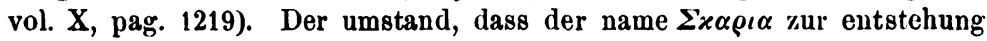
dieser tradition veranlassung gegeben habe, wird hervorgehoben in dem

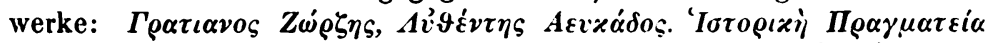

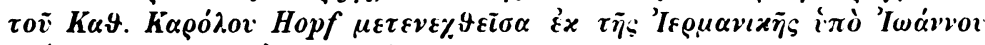

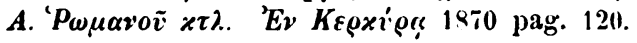


hiertiber so aufgebracht, lass er den echten sohn der königin umbringt und aus dem lande entflieht. Er kommt nun nach Jerusalem, tritt bei Pilatus in dienst und schwingt sich allmählich zu dessen freunde und vertrautem auf. Eirstmals sieht nun Pilatus in einem benachbarten garten sehr schönes obst hängen; da er nach demselben grosses verlangen trägt, dringt Judas sofort in den garten ein, ohne zu wissen, dass derselbe seinem vater Ruben gehört. Mit diesem gerät er dann auch in wortwechsel, als er das obst stehlen will, und erschlägt ihn. Cyborea findet nun ihren gemahl tot in dem garten und zeigt die sache dem Pilatus an. Dieser gibt ihr, um sie für den verlust ihres gatten zu entschädigen, den Judas zam gemahl, aber bald stellt sich heraus, dass er seinen vater gemordet und seine mutter geheiratet hat. Voll reue und sehmerz begibt er sich nun unter die jünger Christi.

Dem verfasser dieser legende kam es zunächst offenbar darauf an, müglichst vicle greuel auf die person des Judas zu häufen, dann aber war er augenscheinlich bestrebt, die gesehichte des Judas mit der legende von Pilatus in zusammenhang zu bringen, die bereits im zwölften jahrhundert vollständig ausgebildet war, während, wie wir gesehen haben, die eiste darstellung der Judaslegende aus dem ende des dreizehnten jahrhunderts stammt. Beide feinde Christi werden als pflegesöhne in eine königliche familie aufgenommen, beide ernorden, und zwar ganz in der nämlichen weise den königsshhn, mit welchem sie gemeinschaftlich erzogen waren und beide missen sich nach diesem morde in ein fremdes land begeben. Auch das verbrechen des selbstmordes wurde dem filatus von der legende aufgebürdet. Wenn in der Judaslegende erzählt wird, wio Jurlas der diener und vertraute des Filatus wurle. so wird fast regelmässig darauf hingewiesen, vie gut beide für cinander passten.

Eigentümlich ist es, dass die legende den Judas als einen s)rössling des stammes Uan darstellt ${ }^{1}$ ), während sonst in den neisten fällen aus seinem beinamen Ischarioth auf seine abvil. V).

3) Isidorus, Allegoriae sacrae scripturae pag. 124 (ed. Arevalo 
stammung vom stamme Isaschar geschlossen wird.1) Diese herleitung vom stamme Dan bezieht sich offenbar auf eine stelle in der rede, mit welcher Jacob kurz vor seinem tode von seinen zwölf söhnen abschied nahm. ${ }^{2}$ ) Hier werden mehrere von den söhnen mit irgend einem tiere verglichen; Juda mit dem löwen, Naphtali mit dem hirsch, Benjamin mit dem wolf, - woher offenbar die jetzt noch unter den Juden viel verbreiteten namen Löb, Hirsch, Wolf etc. ihren ursprung haben; Dan aber wird an dieser stelle mit einer schlange verglichen. ${ }^{3}$ ) Eine behandlung dieses segens Jakobs in lateinischen versen kommt auch in einem gedichte vor, welches Du Méril unter dem titel Cène de Johel veröffentlicht hat. ${ }^{4}$ ) Auf diesen vergleich Dans mit der schlange bezieht es sich offenbar, wenn satan im mittelalter bäufig als ein glied des stammes Dan bezeichnet wird; auch Abraham a Santa Clara spielt hierauf an, wenn er bei erwähnung der abstammung des Judas sagt, dass 'aus der danitischen Schlangenbrut und Zunfft' wol auch der antichrist herstammen werde. ${ }^{5}$ )

In vieler hinsicht ähnelt die jugendgeschichte des Judas auch der jugendgeschichte des Moses, welcher ganz in der nämlichen weise ausgesetzt wird. Auch er wird von einer königlichen frau aufgefunden und an einem fürstenhofe orzogen; auch er muss wegen eines mordes flichtig werden, den er in der ubereilung begangen hat.6) Der gute Abraham a Santa Clara meint auch bei der erzăhlung von der aussetzung des Judas, es sei 'zu schmerzen, dass er in diesem fall dem gerechten Moysi gleichete.' ${ }^{7}$ )

1) Jacobus a Voragine nimmt auch auf diese ansicht rticksicht. Johannes $(13,26)$ nennt den Judas einen sohn Simons. In der franzひsischen verserzählung wird gesagt, der vater des Judas, Ruben, sei aus dem stamme Juda gewesen.

2) Genesis 49. 1-29.

3) Vers 17: Dan wird eine schlange werden auf dem wege und eine otter anf dem steige, und das pferd in die fersen beissen, dass sein reiter zurilckfalle.

4) Poésies latines etc. pag. 97.

) Judas I, 1.

๑) Exodus 2. 3, 10, 12.

7) Judas I, 10. 
Am merkwttrdigsten aber ist an unserer legende ihre ähnlichkeit mit der Oedipussage und mit mehreren anderen legenden des mittelalters, in welchen gleichfalls der elternmord nder ein blutschänderisches verhältnis vorkommt. Hierher gehören die legende von Gregorius auf dem steine, von Paul von Cäsarea, vom heiligen Albanus, vom heiligen elternmörder Julianus Hospitator, ferner die serbische erzählung vom findling Simon und ein italienisches märchen.1) Auch die geschichte von Sir Eglamour of Artois und von Sir Degore, das drama von Walpole: The mysterious mother, sowie mehrere italienische novellen gehören hierher. $\left.{ }^{2}\right)$ Die Oedipussage selbst scheint im mittelalter nicht sehr verbreitet gewesen zu sein. Auf ihr basiert eine lateinische wehklage des Oedipus und ein Roman d'Edipus aus der spätesten zeit des mittelalters. $^{3}$ )

Greith 4) bringt besonders die sagen von Gregorius, Albanus und Julianus mit der Oedipussage in zusammenhang und behauptet, dass die griechische mythe erst durch die christliche behandlung einen versöhnenden abschluss erhalten habe. Comparetti ${ }^{5}$ ) dagegen meint. der grundgedanke sei der dass es kein so schreckliches verbrechen gebe, welches nicht

1) Il figlinolo di germani. Mitgeteilt von Knust in Eberts Jahrbuch YII, 398.

2) Vgl. die einleitung zu der oben erwähnten schrift von D'Ancona, sowie Dunlop-Liebrecht pag. 290.

3) Vgl. ('omparetti, Edipo e ia mitologia comparata. Pisa 1867 jag. 90 und die anzeige von Felix Liebrecht, Göttinger gelehrte anzeigen 1567 pag. 1729. Das lateinische klagelied ist $u$. a. veröffentlicht von ‘chmidt im Philologus XXIII, 515, in vierzciligen strophen abgefasst. Ls stammt spätestens aus dem antang des vierzehnten jahrhunderts, da i) einer handschrift uher Rhythmik, die spätestens aus dieser zeit sammt, der anfang citiert wird (vgl. Berichte der königl. sächs. Gesellschaft der Wissensch. 1871 pag. 71).

4) Greith, Spicilegium Vaticanum. Gregorius auf dem Steine und sein Verhältniss zur mythisch-christlichen Poesie des Mittelalters etc. lag. 137-79; ähnliche ansichten spricht Schreiber aus: Theologische itudien und Kritiken, 1563. Heft 2. Der vermutung Greiths, dass die itreitigkeiten über die lehre von den verbotenen verwantschaftsgraden nit zur entstehung unserer legende beigetragen hätten, tritt Lippold in ceiner weiter unten zu citierenden schrift pag. 34 mit recht entgegen.

s) Comparetti l. c. pag. 87 . 
durch die göttliche gnade gestihnt werden könne. Cholevius bemerkt sehr richtig, dass solche den incest behandelnde dichtungen keineswegs immer aus religiösem ernst entsprangen und dass sowol eine unreine phantasie als das müssige spicl eine unterhaltung darin gefunden hätten, unnatürliche und rätselhafte verwantschaften $\mathrm{zu}$ combinieren.1) Die frommen legendenerzähler verfehlen es in der tat kaum jemals, wenn sich die gelegenheit dazu darbietet, mit seligem behagen dem leser vorzurechnen, dass ein und dieselbe person zugleich gemahl, bruder und sohn derselben frau sei, dass der vater zugleich auch oheim und schwager seines sohnes genannt werden könne u. s. w. ${ }^{2}$ ) In der tat gehören diese legenden zu den widerlichsten auswtichsen der mittelalterlichen literatur und Hartmann muss seine ganze erzählungskunst dazu aufbieten, um uns die ganze jämmerlichkeit seines stoffes für ein par augenblicke rergessen zu lassen. ${ }^{3}$ ) Was nun dic ansicht betrifft, dass die grundidee dieser legenden darin liege, dass selbst die schwerste sünde nach einer aufrichtigen reue von gott verziehen werde. so hätten die legendenerzähler diese idee viel besser an andern stoffen entwickeln können, denn Gregor wie Albanus begehn ganz unfreiwillig die sünden, welche den mittelpunkt der erzählung bilden. Am ehesten kann man sich noch mit der knappen, volkstiimlichen darstellung in der scrbischen erzïhlung versöhnen. ${ }^{\text {) }}$

Von allen diesen legenden zeigt die Judaslegende noch

1) Cholevius, Geschichte der deutschen Poesie nach ihren antiken Elementen 1, pag. $167 \mathrm{f}$.

$\left.{ }^{2}\right)$ Sehr oft erscheinen diese darstellungen solcher verwickelten verwantschaftsverhältnisse auch zur rïtselform zugespitzt, vgl. Dunlop, Geschichte der Prosadichtungen Ubers. von Liebrecht, aum. 268a; Mone, Anzeiger für Kunde der deutschen Vorzeit II, 235. Aehnlich in der legende von Vergogna, cf. d'Ancona l. c. pag. 28, II.

3) Ueher die Gregoriussage cf. Lippold: Ueber die Quelle des Gregorius Har'manns von Aue (Leipziger Doctordissertation) 1869. und Paul in der (in :eitung zu seiner ausgabe des Gregorius. Halle 1573.

4) Mitgeteilt in der sammlung serbischer volkslieder von Talvj; cf. Lippold pag. j.j ff. Ueber die mit dieser sage und der Gregoriuslegende liahe verwante legende von Paul von Cäsarea und über eine dramatische be'landlung der Gregoriuslegende durch den Spanier Juan de Matos Fragoso cf. Köhler in Pfeiffers Germania XV, 284 ff. 
am meisten ähnlichkeit mit der Oedipussage. Sie hat mit ihr den grundgedanken gemeinsam, dass das vorherbestimmte und vorhergeahnte schicksal unabwendbar trotz aller vorsichtsmassregeln eintrifft. In der legende vom heiligen Julianus spielt dieser gedanke allerdings auch eine rolle.1) Hier wird nämlich erzählt, wie Carlos, der vater Julians, Lucinde in Spanien aus einem kloster entfülurt und mit ihr nach Neapel flieht. Dort wird Julian geboren. Als derselbe herangewachsen war und einmal auf der jagd einen hirsch verfolgte, dreht sich dieser plötzlich um und rerkundigt ihm, dass er einstmals seinen vater ermorden werde. In folge dessen kehrt Julian gar nicht mehr nach hause zurtick, sondern flicht nach Spanien, wo er sich mit einem vornehmen fräulein verheiratet. Seine eltern reisen in der ganzen welt herum, um ihren sohn zu suchen, und kommen endlich auch nach Spanien in seine wohnung, als Julian gerade nicht zu hause war. Sie geben sich seiner frau zu erkennen und diese fuhrt zur nachtzeit beide in ihr ehebett. Julian kommt nun spät nach hause und findet schon einen andern mann in seinem bette liegen. Es steigt nun ein böser verdacht in ihm auf und er ermordet seine eltern. Durch ein frommes lehen bijsst er nun bis zum tode seine schuld. Wenn aber auch in dieser sage gleichfalls der gedanke vou der unabwondbarkeit des schicksals ein hauptmotiv ist, so hat sie doch in allen ubrigen zügen gar keine ähnlichkeit mit unserer legende. Die Judaslegende ist die einzige, welche ihrem helden zugleich den incest und den vatermord aufbürdet, und der vatermord des Judas entsteht ebenso wie der vatermord des Oedipus aus einer in ihren grundursachen ganz umbedeutenden zänkerei. Man kann also wol mit bestimmtheit annehmen, dass alle diese motive in unserer legende ursprünglich der Oedipussage entlehnt sind; der umstand, dass diese sage im mittelalter keine volkstümliche ver-

1) Diese legende ist behandelt in zwei spanischen romanzen, abgedruckt bei Duran: Romancero general II, $332 \mathrm{ff}$. Anch diese legende bat in Spanien eine dramatische behandlung gefunden, und zwar in dem Animal Profeta des Lope de Vega, vgl. F. Wolf: Studien zur Geschichte der spanischen und portugiesichen Nationalliteratur pag. 548 . 
breitung hatte, steht dieser annahme nicht im weg, da die Judaslegende ja auch ein wesentlich gelehrtes product ist.

Jedoch hat die Judaslegende offenbar auch einzelne kleine zige von den mehr mit der Gregoriuslegende verwanten sagen entlehnt. Auch Judas wird, ebenso wie Gregorius und Simon, dadurch, dass ihm seine dunkle herkunft zum vorwurf gemacht wird, veranlasst, in die ferne zu fliehen ${ }^{1)}$ und kommt auf diese weise wider mit seiner mutter zusammen. Ein zug, welchen die Gregoriuslegende mit der Oedipussage gemeinsam hat und welcher naturgemäss in der Judaslegende keinen platz finden konnte, ist der, dass beide durch die errettung ihres vaterlandes die hand ihrer mutter erwerben. Die art der aussetzung ist dagegen bei Gregor dieselbe wie bei Judas.2)

In der Brandanuslegende wird erzählt, dass die strafe des Judas in der hölle darin bestand, dass er im meere an einen felsen angeschmiedet war. Dieselbe strafe erduldet bekanntlich auch Gregorius und dies konnte für den verfasser der Judaslegende mit ein grund sein, dem Judas ein ähnliches verbrechen wie dem Gregorius anzudichten.

Die Albanuslegende ist ohne frage von allen diesen legenden die albernste und widerlichste. ${ }^{3}$ ) Albanus ist die frucht

1) Dieser zng findet sich auch in späteren versionen der Oedipussage.

2) Das italienische märchen schliesst sich offenbar an die Gregoriuslegende an, nur dass hier das ganze in bürgerliche verhältnisse übertragen ist. Der bedeutendste unterschied ist der, dass der vater des helden noch zum schlusse in Rom verzeihung erlangt.

3) Einige bemerkungen tuber diese legende gab Køhler in Pfeiffers Germania XIV, 300-304. Lateinisch ist sie behandelt in einer handschr. des Vatican (cod. membr. Urbin. no. 456 unter dem titel vita St. Albini), zuerst erwähnt von Greith (Spicilegium vaticanum pag. 159), dann abgedr. in den Monatsberichten der Berliner akademie 1860 pag. $241 \mathrm{ff}$. mit einigen bemerkungen von Haupt. Ausserdem sind bruchstïcke eines deutschen gedichtes erhalten, herausg. von Lachmann (Berl. ak. 1836). Eine darstellung unserer legende am ende des buchs von Albrecht v. Eyb 'ob einem manne sei zu nemen ein eelich weyb oder nit' ist erwähnt in v. d. Hagens Germania IX, 247. Eine provençalische bearbeitung von Raimon Feraut ist nur aus einer anspielung in dem leben des heil. Honorat von demselben verfasser bekannt, vgl. Raynouard, Lexique roman I, 573: 'cell que volc romanzar la vida Sant Alban.' Zwei spanische romanzen, die denselben gegenstand behandeln, sind abgedruckt bei Duran: Romancero general II, 319, hier wird erzählt, dass der vater der 
eines incestes von vater und tochter. Auch er heiratet seine mutter und ermordet wissentlich seinen vater, als er denselben ' einmal dabei ertappt, wie er diesen incest zum zweiten male begeht. Incest und elternmord finden also hier unter ganz anderen umständen als bei Oedipus und Judas statt. Die einzige ähnlichkeit besteht, wic schon Lippold hervorgehoben hat, darin, dass Albanus ebenso wie Judas und Oedipus in der fremde von einem kinderlosen königspaare an sohnesstatt aufgenommen wird.

Ohne allen zusammenhang mit diesen legenden ist eine erzählung aus der jugend des Judas, welche sich in dem arabischen evangelium von der kindheit Christi findet. Dort wird erzählt, Judas sei als kind vom teufel besessen gewesen und habe jeden, der ihm in den weg. kam, angefallen und gebissen. Seine mutter wollte ihn zu Christo bringen, der damals noch ein knabe war, damit er ihn durch seine wunderkraft heile. Sie fand diesen vor seinem hause spielend; Judas stürzte sofort auf ihn zu und biss ihn in die seite. In demselben augenblicke wich der büse geist in gestalt eines tollen hundes von ihm; Christus aber wurde später, als er gekreuzigt wurde, an derselben stelle von der lanze getroffen, an welcher ihn Judas gebissen hatte. ${ }^{\text {) }}$

Eine ganz isolierte stellung nimmt ferner ein altes englisches gedicht ein, von welchem uns aber nur ein unbedeutendes bruchstuck erhalten ist. Es komint in diesem bruchstück

tochter mit dem tode droht, wenn sie ihm nicht zu willen sein wollte. Die darstellung ist hier sehr schwulstig und bombastisch. Duran vermutet auch hinter der legende eine tiefe sittliche tendenz. Er sagt u. a.: si esta leyenda de San Albano no fuese verdadera y santa, pudiera considerarse como una novela cuyo autor quisó reunir en la persona y vida del Santo todos los crimenes, adulterios, incestos y parricidios que inventó el paganismo griego y atribuyó a los Atridas y a los grandes heroes de sus tiempos histórico-faluulosos. Er erwähnt, dass die legende heute noch in Spanien sehr populär ist; vgl. auch Brunet vol. I s. v. Albanus. - Verwantschaft mit diesen legenden einerseits und der Perseussage anderseits zeigt ein albanesisches märchen, mitgeteilt von Hahn, Albanesische uärchen II, 314, in etwas anderer fassung mitgeteilt von Camarda, cf. Comparetti 1. c. pag. 83.

1) Evangelium infantiae cap. 35. Thilo cod. apocr. pag. 109. 
eine schwester des Judas vor, von welcher sonst nirgends die rede ist; Judas wird von dem Juden Pilatus verftihrt, Christum fur 30 silberlinge $\mathrm{zu}$ verraten. ${ }^{1}$ )

Trotzden nun aber alle möglichen greuel auf die person des Judas gehäuft werden, wird dennoch an der vorstellung festgehalten, dass er auch als apostel gewirkt habe und in besitze der kraft, wunderwerke zu tum, gewesen sei. Hieraus wird nun gefolgert, dass ein geweihter priester, so lange er sich in dicser stellung befindet, alle priesterlichen functionen mit voller giltigkeit ausüben kann und dass in dieser hinsicht seine persönlichen eigenschaften vollständig irrelevant sind.2) $\mathrm{Ob}$ nun aber Christus vorhergewust habe, dass ihn Judas verraten werde und warum er ihn alsdann unter dic apostel aufgenommen habe: auf diese fragen einzugehen, ist hier nicht der ort. Es sei nur erwälnt, dass 'I homas von Aquino der ansicht ist, Christus habe Judas deshall unter die jlinger aufgenommen, weil kein stand ohne schandfleck sei und auch mitten unter den guten eine bosheit lebe. ${ }^{3}$ ) Durch diese auffassung von der stellung des Judas wird man unwillkürlich daran erinnert, dass der verräter Ganelon unter den tapfern, ehrlichen helden Karls des grossen und in mehr scherzhafter, ironischer weise der seneschall Key unter den höfischen und galanten rittern der tafelrunde eine ganz ähnliche stellung einnimmt.

Die höllenstrafen des Judas werden, wie bereits oben angedeutet wurde, besonders ausführlich in der legende vom abt Brandanus behandelt. Dieser findet auf seiner wunderbaren reise eines tages mitten im meere auf einem steine einen mann, weleher an der einen hälfte seines kürpers vom froste und an der audern von der hitze gepeinigt wird. Vor den augen hat er ein tüchlein, wodurch diese ein wenig ror der

1) Abgedr. bei Wright u. Halliwell reliquiae antiquac vol. I, p. 144; bei Mätzner, Sprachproben I, 1 pag. 113.

2) vgl. Zedler, art. Judas. Don Juan Manuel im libro de los estados setzt auch auseinander, wie das beispiel des Judas beweise, dass die schlechtigkeit eines einzelnen der wiirde des ganzen standes keinen eintrag tun könne. (Bibl. d. autores españoles; escr. ant. al siglo XV pag. 354.)

$\left.{ }^{3}\right)$ Judas der Erzschelm. I, pag. 137. 138. 
hitze geschütz.t sind. Er erzählt, er sei Judas, welcher den herrn rerraten habe. Jedoch, sagt er, der verrat wäre ihm wol vergeben worden, weun er sich nicht selbst ermordet hätte. Die qualen, die er auf dem stcine erduldet, seien für ihn jedoch bloss eine sonntagserholung, in der woche werde er mit Pilatus, Herodes, Annas und Kaiphas im höllischen feuer gepeinigt. Das tüchlein, mit welchem er die hitze von den augen abwehrt, hatte er, wie er weiter erzählt, seiner zeit Christo gestohlen und schenkte es später einem aussätzigen, als ihn sein diebstahl gereute. Die eisenhaken, an welchen das tüchlein befestigt ist, hatte er in den tempel gestiftet, um die vorhänge daran aufzuhängen; mit dem stein, auf welchem er sitzt, hatte er in Jerusalem auf der strasse eine den verkehr störende grube ausgefüllt. Als die teufel nun am abend kommen, um Judas wider in das höllische feuer abzufuhren, bewirkt Brandanus durch sein gebet, dass sie ihn noch eine zeit lang in ruhe lassen. Weun (holevius ${ }^{1}$ ) durch diesen let\%teren unstand daran erinnert wird, dass auch Orpheus in der unterwelt durch seinen gesang die qualen des Ixion und des 'Tantalos unterbricht, so ist diese reminiscenz offenbar zu weit hergeholt. In den vielen niederschriften der Brandanuslegende ${ }^{2}$ ) wird diese begegnung des Brandanus mit Judas fast ganz gleichmässig erzählt, nur in der angabe der zeit, welche Judas auf dem steine zubring $\imath$ und in der aufzählung seiner guten handlungen finden sich kleine variationen. Dieser darstellung der höllenqualen des Judas schliesst sich auch der dichter der in leoninischen hexametern abgefassten lebensbeschreibung des Judas an.

In ähnlicher weise wird die bestrafung des Judas in der erzïllung von der reise des Huon von Bordeaux geschildert. Huon findet iu meer auf' der' reise nach Asien in einem strudel einen schwimmer. Dieser erzählt, er sei Judas Ischarioth und sei dazu verdammt, bis in ewigkeit in diesem strudel herungeworfen $\mathrm{zu}$ werden. Der einzige schutz, den er gegen die wut der elemente habe, sei ein kleines stuck tuch, welches

1) Cholevius 1. c. I, 170.

*) cf. Jubinal, la légende latine de St. Brandaine etc. pag. $42 \mathrm{f}$., 150 f. Schröder, Sanct Brandan etc. pag. 29 f., 71 f., $128 \mathrm{f}$. 
er einst bei lebzeiten einem armen schenkte.1) - Von Baudouín de Sebourg wird gleichfalls eine begegnung mit Judas erzählt. Baudouin wird auf einer seefahrt mit dem heiden Poliban weit verschlagen; er gelangt zuerst an das paradies und weiterhin an eine felsige insel, wo sie den ton einer kläglichen stimme vernehmen. Sie treten herzu und bemerken Judas, der ihnen seine höllenqualen in ähnlicher weise schildert, wie sie in der Brandanuslegende erzählt werden. Am samstag und sonntag wird er, wegen einiger guten handlungen, die er im leben begangen hatte, aus der hölle befreit, sonst sitzt er im untersten, dritten grade der hölle. Auf Poliban machen die schrecknisse der hölle einen solchen eindruck, dass er gelobt, christ zu werden (Hist. lit. de la France vol. XXV pag. 573 ff.).

Berthmt ist die grossartige schilderung der höllenqual des Judas in Dantes göttlicher komödie. Er sitzt mit den grösten verbrechern zusammen im tiefsten grunde der hölle. Der dreiköpfige Lucifer hălt ihn, den verräter am himmlischen reiche Christi, mit dem munde seines roten hauptes festgepackt, während Brutus und Cassius, die beiden verräter am weltreiche Cäsars, der eine in seinem schwarzen, der andere in seinem gelben haupte stecken. ${ }^{2}$ )

Eine eigentumliche tradition uber das tun und treiben des Judas im jenseits hat sich in Siebenburgen erhalten. Dort wird erzählt, Judas habe, während Petrus schlief, sonne und mond aus dem himmel gestohlen. Elias erbietet sich nun, ihn zu bezwingen, wird mit blitz und donner ausgerlstet, packt ihn und bindet ihn mit eisenfesseln an eine säule. ${ }^{3}$ )

Bei der kunstlerischen darstellung des Judas bildete sich ebenso wie bei der darstellung anderer biblischer persönlichkeiten ein bestimmter typus aus. Er wird meist als rothaarig dargestellt, wie denn uberhaupt bekanntlich im volksglauben die roten haare als ein böses zeichen gelten.4) Jakob Grimm

1) Dunlop-Liebrecht pag. 128.

2) Inferno cant. XXXIV. Diese schilderung der höllenstrafe des Judas ist bildlich dargestellt von Orcagna in St. Maria Novella in Florenz (cf. Jameson, Sacr. a. leg. art pag. 260).

3) Wilhelm Schmidt, das Jahr und seine Tage in Meinung und Brauch der Romänen Siebenbiirgens. Hermannstadt 1867, pag. 14.

†) Vgl. Du Méril l. c. 324 anm. 
vermutet, dass die rothaarigen wegen der ähnlichkeit ihrer haarfarbe mit der farbe des fuchses in miscredit gekommen seien.' ${ }^{\prime}$ Ausserdem lieben es die klinstler, dem Judas einen prononciert judischen gesichtstypus zu verleihen, was sie bei den ubrigen neutestanicntlichen persönlichkeiten $\mathrm{zu}$ vermeiden suchen. Bekannt ist die anecdote, dass Leonardo da Vinci in seiner weltberühmten darstellung des abendmahls im refectorium des dominikanerklosters zu Mailand bei der ausführung des kopfes des Judas den prior des klosters portraitierte, weil dieser ihn immerfort durch sein drängen auf beschleunigung der arbeit belästigt hatte. Ausserdem wird Judas meist ohne nimbus abgebildet. ${ }^{2}$ ) In einer kirche in Athen ist Judas bei der darstellung der abendmahlscene mit einem schwarzen nimbus abgebildet. ${ }^{3}$ ) In den bühneuanweisungen für die mysterien fand ich nirgends eine andeutúng tiber die tracht des Judas; bei der procession, welche dem Luzerner osterspiele voraufgieng, wurde er wie ein verbrecher in einem karren gefahren.4)

1) Reinhart Fuchs $\mathrm{XXX}$, wo iberhaupt von der büsen bedeutang der roten farbe gehandelt wird. Auch an den roten bart Sibichs wird dort erinnert. Shakespeare (As you like it III, 4) spielt auch auf die rote hasrfarbe des Judas an. Er bezeichnet dies rot als dissembling colour.

2) Fra Angelico pflegt den Judas mit einem nimbus abzubilden, in sinem bilde, das die auszahlung der silberlinge darstellt (Jameson, Sacr. a. eg. art pag. 259) und in einem bilde, den kuss darstellend. Reproduciert bei Jamesun: The history of our lord; 2. ed. London 1865.

3) Didron, Iconographie chrétieune pag. $160 \mathrm{ff}$.

1) Leibing, die Inscenirung des zweitägigen Luzerner Osterspiels lurch Renwart Cysat, pag. 15.

LEIPZIG.

W. CREIZENACH. 